\title{
Distributed Fault Localization in Hierarchically Routed Networks
}

\author{
Malgorzata Steinder and Adarsh Sethi \\ Computer and Information Sciences Department, University of Delaware \\ Newark, DE, USA \\ \{steinder, sethi\}@cis.udel.edu
}

\begin{abstract}
Probabilistic inference was shown effective in non-deterministic diagnosis of end-to-end service failures. To overcome the exponential complexity of the exact inference algorithms in fault propagation models represented by graphs with undirected loops, Pearl's iterative algorithms for polytrees were used as an approximation schema. The approximation made it possible to diagnose end-toend service failures in network topologies composed of tens of nodes. This paper proposes a distributed algorithm that increases the admissible network size by an order of magnitude. The algorithm divides the computational effort and system knowledge among multiple, hierarchically organized managers. The cooperation among managers is illustrated with examples, and the results of a preliminary performance study are presented 1
\end{abstract}

\section{Introduction}

End-to-end network service failure diagnosis [15,17] is a sub-task of fault localization [79.19] that isolates host-to-host services responsible for availability or performance problems associated with a communication between end-hosts. In [1617], probabilistic inference was applied to provide a non-deterministic solution to this problem. The probabilistic fault propagation model representing the problem of end-to-end service failure diagnosis is a bipartite directed graph, which contains undirected loops. To overcome the exponential computational complexity required by the exact inference algorithms in graphs with loops, Pearl's iterative algorithms for polytrees were used as an approximation schema [17]. The algorithm introduced in [17] diagnoses end-to-end service failures in network topologies composed of tens of nodes, but it does not scale well to topologies composed of hundreds of nodes.

This paper expands on the iterative algorithm proposed in [17] and introduces its distributed version that increases the admissible network size by an order of magnitude. The algorithm divides the computational effort and system knowledge involved in end-toend service failure diagnosis among multiple, hierarchically organized managers. Each

\footnotetext{
${ }^{1}$ Prepared through collaborative participation in the Communications \& Networks Consortium sponsored by the U. S. Army Research Lab under the Collaborative Technol. Alliance Program, Cooperative Agreement DAAD19-01-2-0011. The U. S. Government is authorized to reproduce and distribute reprints for Government purposes notwithstanding any copyright notation thereon.
} 
manager is responsible for fault localization within the network domain it governs, and reports to the higher-level manager which oversees and coordinates the fault localization process of multiple domains. With this organization, the technique is suitable for endto-end service failure diagnosis in hierarchically routed networks.

The paper is structured as follows. In Section 2, we give background information on applying probabilistic inference to the problem of end-to-end service failure diagnosis. Section 3 introduces the distributed version of the algorithm. Section 4 presents illustrative examples of cooperation among managers. In Section 5, preliminary results of a simulation study are presented. The related work is described in Section 6

\section{Centralized End-to-End Service Failure Diagnosis}

End-to-end service failure diagnosis [14] uses a directed bipartite graph as a fault propagation model, whose parentless vertices represent host-to-host (link) failures, and childless vertices represent the resultant end-to-end service (path) failures. Host-to-host and end-to-end service failures are considered faults and symptoms, respectively. The diagnosis of performance-related or upper-layer problems requires a probabilistic fault model, in which parentless vertices (root causes) are labeled with independent failure probabilities and the directed edges are weighted with conditional probabilities representing the strengths of causal influences between host-to-host and end-to-end service failures. Typically [9.10,17], a noisy-OR probability model [12] is used.

Diagnosing end-to-end service failures may be mapped into the problem of finding the most probable explanation of the observed evidence in belief networks [12]. While the problem is known to be NP-hard [2], a polynomial-time inference algorithm was proposed for a restricted class of belief networks represented by singly-connected graphs [12]. The algorithm is commonly referred to as Pearl's iterative belief propagation. In [17], Pearl's algorithm is used as an approximation schema for end-to-end service failure diagnosis based on fault propagation models with loops.

Iterative belief propagation utilizes a message-passing schema, in which the belief network vertices exchange $\lambda_{X}$ and $\pi_{X}$ messages, which denote posterior and prior belief with respect to node $X$ [12]. The belief propagation algorithm in polytrees starts from evidence vertices and propagates the changed belief along the graph edges by computing $\operatorname{bel}(x), \lambda_{X}\left(v_{i}\right)$ 's and $\pi_{X}\left(u_{i}\right)$ 's [12] in every visited vertex. The technique introduced in [17] (Algorithm 1) adapts the iterative belief propagation algorithm to the problem of fault localization with fault models represented by bipartite graphs with undirected loops. In this event-driven technique, one traversal of the graph is performed for every observed symptom. The set of all observed symptoms is denoted by $\mathcal{S}_{O}$.

\section{Algorithm 1 (MPE through iterative belief updating)}

\section{Inference iteration starting from vertex $Y_{i}$ :}

let $o$ be the breadth-first order starting from $Y_{i}$

for all vertices $X$ such as $X$ is not an unobserved path vertex, along ordering $o$

compute $\lambda_{X}\left(v_{j}\right)$ for all $X$ 's parents, $V_{j}$, and for all $v_{j} \in\{0,1\}$

compute $\pi_{U_{i}}(x)$ for all $X$ 's children, $U_{i}$, and for all $x \in\{0,1\}$

\section{Symptom analysis phase:}


for every symptom $S_{i} \in \mathcal{S}_{O}$ run inference iteration starting from $S_{i}$ compute bel $\left(v_{i}\right)$ for every vertex $V_{i}, v_{i} \in\{0,1\}$

\section{Fault selection phase:}

while $\exists$ link vertex $V_{j}$ for which bel $(1)>0.5$ and $S_{O} \neq \emptyset$ do

take $V_{j}$ with the greatest bel(1) and mark it as observed to have value of 1

run inference iteration starting from $V_{j}$

remove all $S_{i}$ such that $V_{j}$ may cause $S_{i}$ from $S_{O}$

compute bel $\left(v_{i}\right)$ for every vertex $V_{i}, v_{i} \in\{0,1\}$

It was shown through simulation that the algorithm offers close to the optimal accuracy [17]. The approximation significantly improves the feasibility of fault localization over the exact (but exponential) algorithm and allows the end-to-end service failure diagnosis to be efficiently performed in networks composed of tens of nodes. Additionally [16], the algorithm does not require the accurate knowledge of conditional probability distribution being able to retain high accuracy when a few confidence intervals are used instead of the exact conditional probabilities. It is also resilient to lost and spurious symptoms and allows positive symptoms to be incorporated without increasing the algorithm's computational complexity [16].

\section{Distributed End-to-End Service Failure Diagnosis}

In this paper, we propose a distributed fault management technique of end-to-end service failure diagnosis, which takes advantage of the domain semantics of real-life communication systems. The management domains considered by the technique correspond to administrative or routing network domains. We adopt the hierarchical organization of the management system. Although multiple levels of hierarchy are possible, we will describe a two level management system in which domain managers (DM) report to the global manager (GM) overseeing the entire network.

We introduce the following notation.

- $\mathcal{D}_{1}, \ldots, \mathcal{D}_{n}$ - The set of management domains within the managed system

- $D M_{i}$ - Manager of domain $\mathcal{D}_{i}$

- $d_{i}-$ A unique identifier of domain $\mathcal{D}_{i}$, e.g., a network IP address and mask.

- $n_{k}-\mathrm{A}$ unique identifier of a node in domain $\mathcal{D}_{i}$, e.g., its IP host address.

- $d_{i} \cdot n_{k}$ - Network-wide unique identifier of node $n_{k} \in \mathcal{D}_{i}$.

- $d_{i} . n_{k} \rightarrow d_{j} . n_{l}-\mathrm{A}$ directed link from node $d_{i} . n_{k}$ to node $d_{j} . n_{l}$.

- $d_{j_{1}} \cdot n_{p_{1}} \stackrel{*}{\rightarrow} d_{j_{m}} \cdot n_{p_{m}}-$ A directed, possibly multihop path from node $d_{j_{1}} \cdot n_{p_{1}}$ to node $d_{j_{m}} . n_{p_{m}}$ consisting of links $d_{j_{1}} . n_{p_{1}} \rightarrow d_{j_{2}} . n_{p_{2}}, \ldots, d_{j_{m-1}} . n_{p_{m-1}} \rightarrow d_{j_{m}} . n_{p_{m}}$.

- $s_{v}: d_{i} \cdot n_{k} \stackrel{*}{\rightarrow} d_{j} . n_{l}$ - A symptom associated with path $d_{i} \cdot n_{k} \stackrel{*}{\rightarrow} d_{j} \cdot n_{l}$

- $f_{w}: d_{i} \cdot n_{k} \rightarrow d_{j} . n_{l}-\mathrm{A}$ fault associated with link $d_{i} . n_{k} \rightarrow d_{j} . n_{l}$

- $d_{i} \stackrel{*}{\rightarrow} d_{j}$ - The set of all paths which begin in domain $\mathcal{D}_{i}$ and end in domain $\mathcal{D}_{j}$, i.e., $d_{i} \stackrel{*}{\rightarrow} d_{j}=\left\{d_{i} . n_{k} \stackrel{*}{\rightarrow} d_{j} . n_{l} \mid n_{k} \in \mathcal{D}_{i}\right.$ and $\left.n_{l} \in \mathcal{D}_{j}\right\}$.

- $s_{z}: d_{i} \stackrel{*}{\rightarrow} d_{j}$ - A symptom associated with the set of paths $d_{i} \stackrel{*}{\rightarrow} d_{j}$, which indicates that at least one $s_{v}: d_{i} . n_{k} \stackrel{*}{\rightarrow} d_{j} . n_{l}$ occurred such that $n_{k} \in \mathcal{D}_{i}$ and $n_{l} \in \mathcal{D}_{j}$.

In a multi-domain environment, a fault in one domain may cause symptoms in other domains. For example, a fault causing a failure of path $d_{i} . n_{k} \stackrel{*}{\rightarrow} d_{i} . n_{l}$, in domain $\mathcal{D}_{i}$ 
may be observed as a failure of an inter-domain path $d_{j_{1}} \cdot n_{p_{1}} \stackrel{*}{\rightarrow} d_{j_{m}} . n_{p_{m}}$, such that $d_{i} . n_{k}, d_{i} . n_{l} \in\left\{d_{j_{1}} . n_{p_{1}}, \ldots, d_{j_{m}} . n_{p_{m}}\right\}$ and $d_{i} . n_{k}$ precedes $d_{i} . n_{l}$. Such a path will be referred to as an intra- $\mathcal{D}_{i}$ segment of path $d_{j_{1}} \cdot n_{p_{1}} \stackrel{*}{\rightarrow} d_{j_{m}} \cdot n_{p_{m}}$.

In the proposed technique, an inter-domain symptom $s_{t_{1}}: d_{j_{1}} \cdot n_{p_{1}} \stackrel{*}{\rightarrow} d_{j_{m}} \cdot n_{p_{m}}$ is handled by the GM, which is able to identify intra-domain path segments which might have caused $s_{t_{1}}$. GM delegates the task of intra-domain path segments' diagnosis to the corresponding domain managers. Thus, after symptom $s_{t_{1}}$ is observed, GM will delegate the diagnosis of path segment $d_{i} . n_{k} \stackrel{*}{\rightarrow} d_{i} . n_{l}$ to $D M_{i}$. It does so by simply creating and reporting symptom $s_{t_{2}}: d_{i} . n_{k} \stackrel{*}{\rightarrow} d_{i} . n_{l}$ to $D M_{i}$. With every such symptom a high level of uncertainty is associated: since $s_{t_{1}}$ might have been caused by path segments located in domains other than $\mathcal{D}_{i}$, symptom $s_{t_{2}}$ passed to $D M_{i}$ is likely to be spurious. While forwarding $s_{t_{2}}$ to $D M_{i}$ the GM includes the value of the belief with which the symptom should be considered spurious in $\mathcal{D}_{i}, p_{s}\left(s_{t_{2}}\right)$, and the information on a path between which two domains the failure occurred. The $D M_{i}$ correlates symptoms received from the GM with those reported internally in domain $\mathcal{D}_{i}$.

A failure of an inter-domain path $d_{j_{1}} \cdot n_{p_{1}} \stackrel{*}{\rightarrow} d_{j_{m}} . n_{p_{m}}$ may also be caused by a failure of an inter-domain link $d_{j_{s}} . n_{p_{s}} \rightarrow d_{j_{s+1}} . n_{p_{s+1}}$, such that $d_{j_{s}} \cdot n_{p_{s}}, d_{j_{s+1}} . n_{p_{s+1}} \in$ $\left\{d_{j_{1}} . n_{p_{1}}, \ldots, d_{j_{m}} \cdot n_{p_{m}}\right\}$. Failures of inter-domain links have to be isolated by the GM, since no domain manager has sufficient knowledge about inter-domain connectivity to make this determination. During the process of diagnosing inter-domain symptoms, which might have been caused by both an inter-domain link failure and an intra-domain path-segment failure, the GM must collaborate with the domain managers. The higher the probability that a fault occurred in domain $\mathcal{D}_{i}$, which might have caused the failure of an intra- $\mathcal{D}_{i}$ segment of an inter-domain path, the lower the probability that the inter-domain path failure has been caused by any inter-domain link. Thus, before reporting an interdomain link as a possible cause of a failure of inter-domain path $d_{j_{1}} \cdot n_{p_{1}}{ }^{*} d_{j_{m}} \cdot n_{p_{m}}$, the GM requests from the manager of each domain $\mathcal{D}_{i}$ traversed by path $d_{j_{1}} . n_{p_{1}} \stackrel{*}{\rightarrow} d_{j_{m}} . n_{p_{m}}$, the failure probability of an intra- $\mathcal{D}_{i}$ path segment of $d_{j_{1}} \cdot n_{p_{1}} \stackrel{*}{\rightarrow} d_{j_{m}} . n_{p_{m}}$, which is independent of any inter-domain symptom observations. To ensure the independence, the intra-domain path-failure probability is calculated by $D M_{i}$ based solely on the symptoms observed by $D M_{i}$, excluding those that $D M_{i}$ receives from GM. As new evidence, inaccessible to GM, is observed and analyzed within $\mathcal{D}_{i}$, the value of this probability changes.

\subsection{Distributed Fault Propagation Model}

In a distributed technique, the responsibility for maintaining the fault localization model is shared among DMs and the GM. Domain managers maintain fault propagation models for the domains they manage. A fault propagation model built by manager $D M_{i}$ represents causal relationships among path and link services provided within domain $\mathcal{D}_{i}$. Such a model may be built in advance or dynamically extended as symptoms related to particular paths are observed. For every monitored path $d_{i} . n_{p_{1}} \stackrel{*}{\rightarrow} d_{i} . n_{p_{m}}, D M_{i}$ 's model contains vertices $V_{p}, V_{l_{1}}, \ldots$, and $V_{l_{m-1}}$ labeled $s_{p}: d_{i} . n_{p_{1}} \stackrel{*}{\rightarrow} d_{i} . n_{p_{m}}, f_{l_{1}}: d_{i} . n_{p_{1}} \rightarrow d_{i} . n_{p_{2}}$, $\ldots, f_{l_{m-1}}: d_{i} \cdot n_{p_{m-1}} \rightarrow d_{i} . n_{p_{m}}$, respectively. It also contains causal edges, each originating from one of $V_{l_{1}}, \ldots$, and $V_{l_{m-1}}$, which end at $V_{p}$. Link vertices are also labeled 
with prior failure probabilities, and causal edges are weighted with the probability of the causal influence taking place.

As an example, consider a threedomain network presented in Fig. 1, To diagnose symptom $s_{4}: 2.17 \stackrel{*}{\rightarrow} 2.19$, given path $2.17 \stackrel{*}{\rightarrow} 2.19$ consists of links $2.15 \rightarrow 2.19$ and $2.17 \rightarrow 2.15, \quad D M_{2}$ creates vertices labeled $s_{4}: 2.17 \stackrel{*}{\rightarrow} 2.19$, $f_{2}: 2.15 \rightarrow 2.19$ and $f_{4}: 2.17 \rightarrow 2.15$, and connects vertices $f_{2}: 2.15 \rightarrow 2.19$ and $f_{4}: 2.17 \rightarrow 2.15$ to vertex $s_{4}: 2.17 \stackrel{*}{\rightarrow} 2.19$. $D M_{2}$ 's belief network presented in Fig.2 models fault propagation for symptoms $\quad s_{1}: 2.15 \stackrel{*}{\rightarrow} 2.16, \quad s_{2}: 2.15 \stackrel{*}{\rightarrow} 2.19$, $s_{3}: 2.15 \stackrel{*}{\rightarrow} 2.18$, and $s_{4}: 2.17 \stackrel{*}{\rightarrow} 2.19$.

This model is dynamically extended as new symptoms are received from the GM. Recall that the GM delegates a part of the fault diagnosis task initiated by an interdomain symptom to DMs of domains traversed by the corresponding path. Thus, for an inter-domain path $d_{j_{1}} \cdot n_{p_{1}} \stackrel{*}{\rightarrow} d_{j_{m}} . n_{p_{m}}$, which includes path segment $d_{i} \cdot n_{k} \stackrel{*}{\rightarrow} d_{i} . n_{l}$, the GM forwards to $D M_{i}$ the following information: symptom $s_{t}: d_{i} \cdot n_{k} \stackrel{*}{\rightarrow} d_{i} \cdot n_{l}$, the probability that $s_{t}$ is spurious in $\mathcal{D}_{i}, p_{s}\left(s_{t}\right)$, and a pair $\left(d_{j_{1}}, d_{j_{m}}\right) . D M_{i}$ creates (if it does not yet exist) a new vertex $V_{p}$ labeled with $\overline{s_{p}}: d_{j_{1}} \stackrel{*}{\rightarrow} d_{j_{m}}$ and then creates a causal edge from $V_{p}$ to vertex $V_{t}$ labeled with $s_{t}: d_{i} \cdot n_{k} \stackrel{*}{\rightarrow} d_{i} . n_{l}$. Vertex $V_{p}$, which represents all possible causes of symptom $s_{t}$ that are external to $\mathcal{D}_{i}$, is additionally labeled with $p_{s}\left(s_{t}\right)$. The edge from $V_{p}$ to $V_{t}$ is assigned weight 1.0 . For every symptom vertex the following function is defined: state-of: $V \rightarrow\{$ UNOBSERVED, OBSERVED-INTERNAL, OBSERVED-EXTERNAL\}.

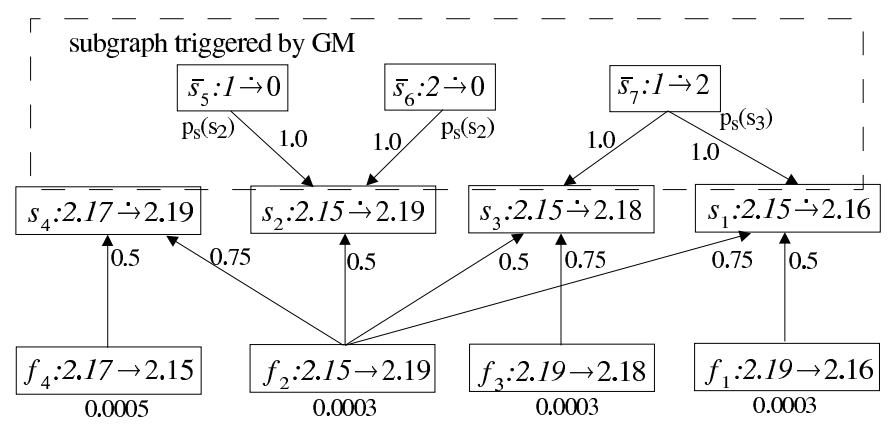

Fig. 2. $D M_{2}$ 's belief network

As an example, consider link $2.15 \rightarrow 2.19$ in domain $\mathcal{D}_{2}$ in Fig. 1 , which belongs to the shortest path between domains $\mathcal{D}_{1}$ and $\mathcal{D}_{0}$. Its failure may cause a failure of inter-domain 
path $1.22 \stackrel{*}{\rightarrow} 0.28$ consisting of links $1.22 \rightarrow 1.20,1.20 \rightarrow 1.24,1.24 \rightarrow 2.15,2.15 \rightarrow 2.19$, $2.19 \rightarrow 0.25$, and $0.25 \rightarrow 0.28$. The GM identifies the following possible causes of the symptom: intra- $\mathcal{D}_{1}$ path segment $1.22 \stackrel{*}{\rightarrow} 1.24$, link $1.24 \rightarrow 2.15$, intra- $\mathcal{D}_{2}$ path segment $2.15 \stackrel{*}{\rightarrow} 2.19$, link $2.19 \rightarrow 0.25$, and intra- $\mathcal{D}_{0}$ path segment $0.25 \stackrel{*}{\rightarrow} 0.28$. $D M_{2}$ receives from the GM symptom $s_{2}: 2.15 \stackrel{*}{\rightarrow} 2.19, p_{s}\left(s_{2}\right)$, and a pair $(1,0)$. It creates a vertex labeled $\overline{s_{5}}: 1 \stackrel{*}{\rightarrow} 0$ and connects it to the vertex labeled $s_{2}: 2.15 \stackrel{*}{\rightarrow} 2.19$ (Fig. 2). The failure of link $2.15 \rightarrow 2.19$ may also cause communication problems from domain $\mathcal{D}_{1}$ to some nodes in $\mathcal{D}_{2}$, and from some nodes in $\mathcal{D}_{2}$ to domain $\mathcal{D}_{0}$. The $D M_{2}$ fault propagation model presented in Fig. 2 includes vertices and edges which are created when failures of paths $1.22 \stackrel{*}{\rightarrow} 0.28,1.23 \stackrel{*}{\rightarrow} 2.18,2.15 \stackrel{*}{\rightarrow} 0.28$, and $1.20 \stackrel{*}{\rightarrow} 2.16$ occur.

A fault propagation model built by the global manager is concerned with connectivity among domains rather than particular nodes. It contains three types of vertices: those representing inter-domain links, inter-domain path sets, and domains. Suppose connectivity between domains $\mathcal{D}_{i}$ and $\mathcal{D}_{j}$ is provided using an inter-domain link $d_{j_{s}} . n_{p_{s}} \rightarrow d_{j_{t}} . n_{p_{t}}$ and intra-domain path segment $d_{u} \cdot n_{k} \stackrel{*}{\rightarrow} d_{u} . n_{l}$. The model should contain a vertex $V_{p}$ labeled with $s_{p}: d_{i}{ }^{*} \rightarrow d_{j}$, which represents the set of all paths that begin in $\mathcal{D}_{i}$ and end in $\mathcal{D}_{j}$, vertex $V_{l}$ labeled with $s_{l}: d_{j_{s}} . n_{p_{s}} \rightarrow d_{j_{t}} . n_{p_{t}}$, and vertex $V_{d}$ representing domain $\mathcal{D}_{u}$ labeled with $D: d_{u}$. The causal edges point from vertices $V_{l}$ and $V_{d}$ to vertex $V_{p}$. Vertex $V_{d}$ is additionally labeled with prior failure probability equal to 1.0. The edge between $V_{d}$ and $V_{p}$ is labeled with $p_{c}\left(d_{u}, d_{i}, d_{j}\right)$, i.e., the probability that as a result of faults in $\mathcal{D}_{u}$, any intra- $\mathcal{D}_{u}$ path segment is affected that belongs to at least one path in the path-set $d_{i} \stackrel{*}{\rightarrow} d_{j}$. Observe that each vertex labeled with $d_{i} \stackrel{*}{\rightarrow} d_{j}$ in GM's model is connected to at least two vertices representing a domain, i.e., these labeled with $D: d_{i}$ and $D: d_{j}$. Similarly to DMs, GM provides function state-of: $V \rightarrow\{$ UNOBSERVED, OBSERVED\}, which is defined for each symptom vertex. Fig. 3 presents a belief network created by a global manager of the network in Fig. 1

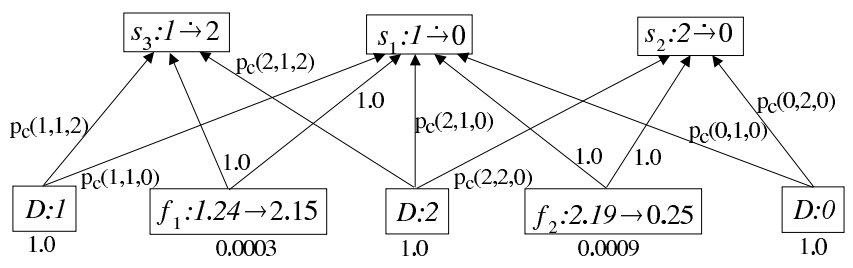

Fig. 3. GM's belief network

\subsection{Global Manager's Fault Localization Algorithm}

We begin describing the distributed fault localization algorithm proposed in this paper by presenting the algorithm executed by the global manager (Algorithm 2). The GM's algorithm is composed of three phases, which may be interleaved. However, for the sake of clarity, they are presented as separate components. 
Model synchronization phase initializes or adjusts conditional probabilities assigned to the causal edges between vertices representing domains and inter-domain paths (Section 3.1). This phase must be first executed when the model is initialized and then repeated before final fault selection is made. The purpose of the repeated model synchronization is to update the values of the conditional probabilities assigned to edges between domain and path vertices, which have changed during fault localization process as a result of intra-domain symptoms analysis. When model synchronization is repeated in the fault selection phase, the for loop iterates only through observed inter-domain path vertices. (Unobserved symptom nodes do not propagate evidence.)

Symptom analysis phase, given an inter-domain symptom, performs one iteration of probabilistic inference proposed in Algorithm 1 1 using the GM's internal fault propagation model. Then, through reporting a symptom, it requests that managers of domains traversed by the inter-domain path perform the same operation using their internal models based on symptoms received from the GM. Observe that in GM's model, vertex $V_{p}$ labeled with $s_{p}: d_{i} \stackrel{*}{\rightarrow} d_{j}$ is assigned the value of 1 as soon as the first symptom referring to a path belonging to the set $d_{i} \stackrel{*}{\rightarrow} d_{j}$ is observed. Subsequent failures of paths from this set are ignored by the GM, which significantly reduces the amount of computation performed by the GM. Also observe that intra-domain path segments of an inter-domain path are easy to determine by scanning the sequence of inter-domain links used to provide connectivity from the domain of origin to the destination domain.

\section{Algorithm 2 (Global Manager's Fault Localization Algorithm)}

\section{Model synchronization phase:}

for every vertex $V_{p}$ labeled with $d_{i} \stackrel{*}{\rightarrow} d_{j}$

for every vertex $V_{d}$ labeled with $D: d_{u}$ such that $V_{d}$ and $V_{p}$ are connected obtain $p_{c}\left(d_{u}, d_{i}, d_{j}\right)$ and label edge $\left(V_{d}, V_{p}\right)$ with $p_{c}\left(d_{u}, d_{i}, d_{j}\right)$.

run inference iteration starting from $V_{d}$

\section{Symptom analysis phase:}

for every observed symptom $s_{u}: d_{i} . n_{k_{i}} \stackrel{*}{\rightarrow} d_{j} . n_{k_{j}}$ such that $d_{i} \neq d_{j}$

let $V_{p}$ be the vertex labeled with $s_{p}: d_{i} \stackrel{*}{\rightarrow} d_{j}$

if state-of $\left(V_{p}\right)=$ UNOBSERVED

set state-of $\left(V_{p}\right)=$ OBSERVED and $V_{p}=1$

run inference iteration starting from $V_{p}$

for every intra-domain path segment of $d_{i} \cdot n_{k_{i}} \stackrel{*}{\rightarrow} d_{j} \cdot n_{k_{j}}, d_{u} . n_{m} \stackrel{*}{\rightarrow} d_{u} . n_{l}$

create $s_{t}: d_{u} \cdot n_{m} \stackrel{*}{\rightarrow} d_{u} \cdot n_{l}$ and calculate $p_{s}\left(s_{t}\right)$

forward $\left\{s_{t}, p_{s}\left(s_{t}\right),\left(d_{i}, d_{j}\right)\right\}$ to $D M_{u}$

\section{Fault selection phase:}

run model synchronization phase for only those path-vertices $V_{p}$

for which state-of $\left(V_{p}\right)=$ OBSERVED

compute bel $\left(v_{i}\right)$ for every vertex $V_{i}, v_{i} \in\{0,1\}$

run fault selection phase of Algorithm $\square$ 


\subsection{Domain Manager's Fault Localization Algorithm}

Domain manager in domain $\mathcal{D}_{i}$ receives symptoms from two sources: from nodes in $\mathcal{D}_{i}$ and from the global manager. When a symptom from the global manager is observed, $D M_{i}$ first updates the model as described in Section 3.1, Then it marks the symptom vertex as observed outside domain $\mathcal{D}_{i}$ and runs one inference iteration starting from this vertex. When an internal symptom is observed it overrides any previous external observations of the same symptom. The causal relationships between the symptom vertices and vertices representing causes outside of $\mathcal{D}_{i}$ are removed, as the symptom is now known to have been caused by a fault in domain $\mathcal{D}_{i}$. Until the internal symptom is cleared, no future external observations of the same symptom are taken into account. In addition, $D M_{i}$ calculates $p_{c}\left(d_{i}, d_{j}, d_{u}\right)$ required by the GM in model synchronization phase of its algorithm (Algorithm 2).

\section{Algorithm 3 (Domain Manager's Fault Localization Algorithm for Domain $\mathcal{D}_{i}$ )}

\section{Symptom analysis phase:}

for every observed symptom $s_{p}: d_{i} . n_{k} \stackrel{*}{\rightarrow} d_{i} . n_{l}$

let $V_{p}$ be the vertex labeled with $s_{p}: d_{i} \cdot n_{k} \stackrel{*}{\rightarrow} d_{i} . n_{l}$

if $s_{p}$ is received from GM in message $\left\{s_{p}, p_{s}\left(s_{p}\right),\left(d_{u}, d_{j}\right)\right\}$

if state-of $\left(V_{p}\right)=$ OBSERVED-INTERNAL then continue $/ *$ ignore $s_{p} * /$

assign weight $p_{s}\left(s_{p}\right)$ to edge $\left(V_{s}, V_{p}\right)$, where $V_{s}$ is labeled with $s_{s}: d_{u} \stackrel{*}{\rightarrow} d_{j}$ set state-of $\left(V_{p}\right)=$ OBSERVED-EXTERNAL and $V_{p}=1$

run inference iteration starting from $V_{p}$

else $/ * s_{p}$ is internal */

assign weight 0 to every edge $\left(V_{s}, V_{p}\right)$, where $V_{s}$ is not a link vertex in $\mathcal{D}_{i}$ set state-of $\left(V_{p}\right)=$ OBSERVED-INTERNAL and $V_{p}=1$

run inference iteration starting from $V_{p}$

Fault selection phase: identical to Algorithm $\square$

\section{Examples}

In this section, we illustrate the algorithms proposed in this paper with examples using the network presented in Fig. 1. We also assume that fault localization models of the GM and $D M_{2}$ are those presented in Figs. 3 and 2.

\subsection{Inter-domain Link Failure Example}

In the first example, we consider a scenario in which a fault occurs in inter-domain link $1.24 \rightarrow 2.15$. As a result, inter-domain symptoms are observed indicating failures of paths $1.20 \stackrel{*}{\rightarrow} 2.16,1.21 \stackrel{*}{\rightarrow} 0.26$, and $1.23 \stackrel{*}{\rightarrow} 2.19$. When the failure of path $1.20 \stackrel{*}{\rightarrow} 2.16$ is observed, the GM runs one iteration of belief updating starting from node labeled $s_{3}: 1 \stackrel{*}{\rightarrow} 2$. This process identifies link $1.24 \rightarrow 2.15$ as a possible cause of the observed path failure. At the same time, the GM delegates the diagnosis of intra-domain path segments which may also be responsible for the failure of $1.20 \rightarrow 2.16$ to domain managers $D M_{1}$ and 
$D M_{2}$ by sending messages $\{1.20 \stackrel{*}{\rightarrow} 1.24,0.003,(1,2)\}$ and $\{2.15 \stackrel{*}{\rightarrow} 2.16,0.003,(1,2)\}$, respectively. Table 1 presents the list of inter- and intra-domain links whose bel(1) exceeds 0.1 after this operation. It shows that neither $D M_{1}$ nor $D M_{2}$ is able to single out a fault meeting this criterion after the first symptom observation. The third symptom $(1.23 \stackrel{*}{\rightarrow} 2.19)$ is ignored by the GM as it belongs to the same path set as one of the symptoms previously analyzed $(1.20 \stackrel{*}{\rightarrow} 2.16)$. In this example, the model synchronization phase does not contribute any changes to the results of GM's fault diagnosis process, since no intra-domain symptoms have been observed.

Table 1. Fault diagnosis process of fault $f_{1}: 1.24 \rightarrow 2.15$

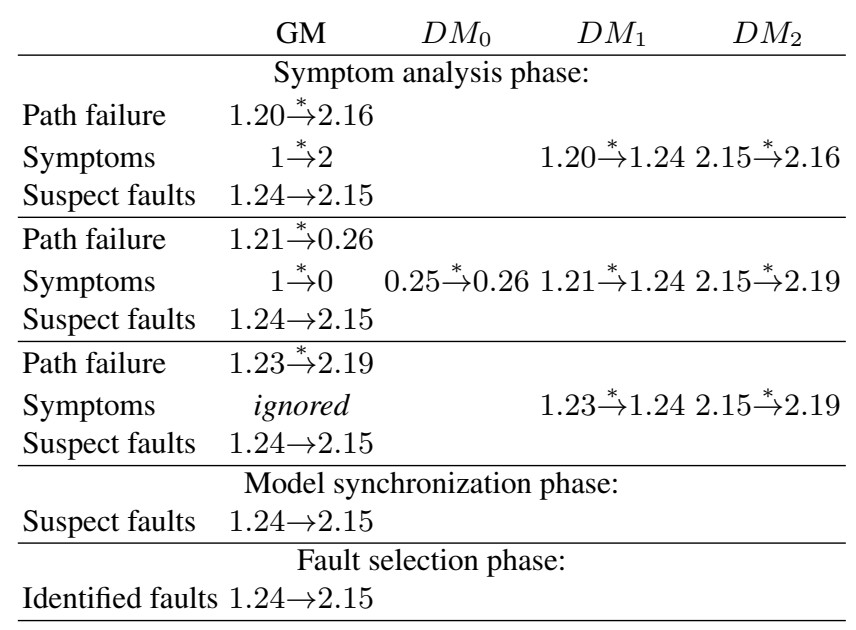

\subsection{Intra-domain Link Failure}

In the second example, we consider a scenario in which a fault occurs in intra-domain link $2.15 \rightarrow 2.19$. Since this link is used by the backbone route between domains 1 and 0 , interdomain symptoms may be generated as a result of the failure. The complete sequence of symptoms generated in the scenario and their diagnosis process are presented in Table 2] Before the model synchronization phase, confidence associated with failures of links $1.24 \rightarrow 2.15,2.19 \rightarrow 0.25$, and $2.15 \rightarrow 2.19$, is $0.29,0.80$, and 0.99 , respectively. Thus, without this phase, two faults would be chosen by the algorithm: $2.19 \rightarrow 0.25$, and $2.15 \rightarrow 2.19$. Updating the model with the information learned by $D M_{2}$ from the internal symptoms it observed allows fault $2.19 \rightarrow 0.25$ to be eliminated.

\section{Preliminary Simulation Study}

This section presents the preliminary results of a simulation study designed to verify concepts in this paper. We use Brite network topology generator [11] to build random, 
Table 2. Fault diagnosis process of fault $f_{2}: 2.15 \rightarrow 2.19$

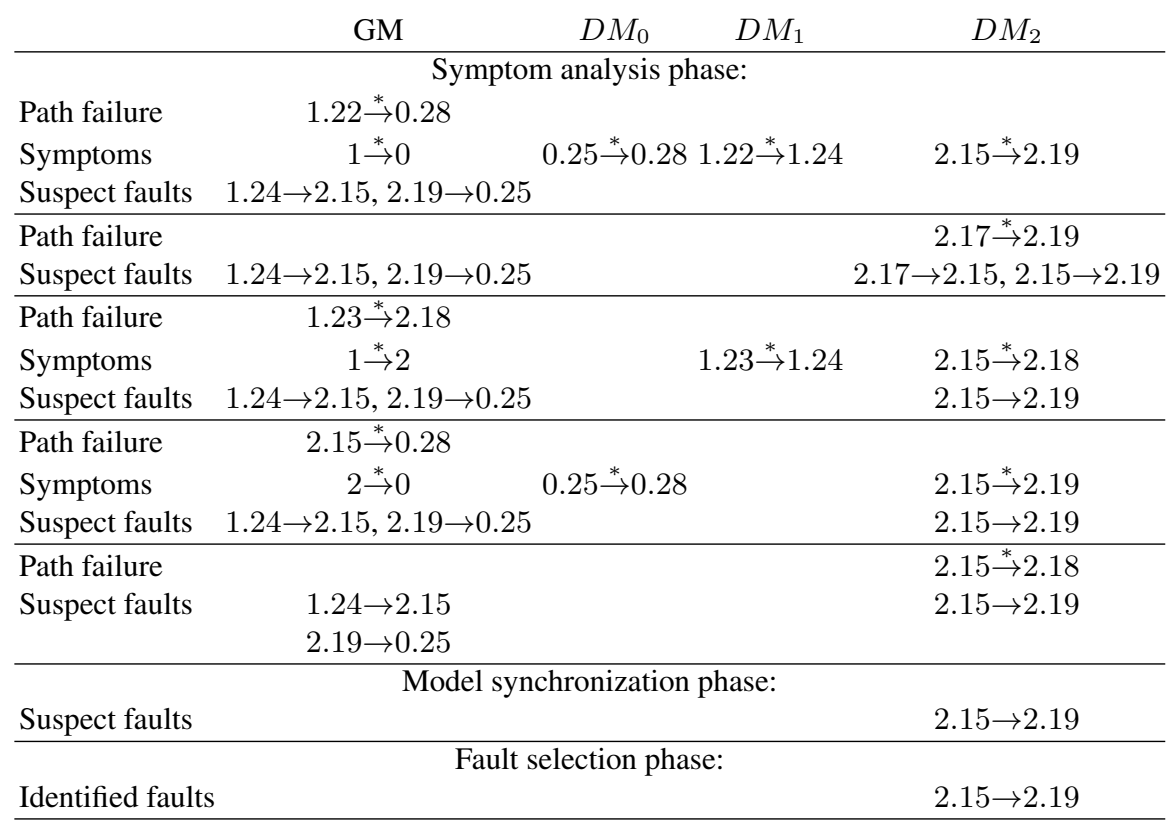

two-level network topologies similar to those of the Internet. Then we build a belief network representing relationships among end-to-end and hop-to-hop services in this communications network assuming a shortest-path routing algorithm. In the belief network, prior link failure probabilities are randomly generated as uniformly distributed random variables over the range $(0.0001,0.001)$. Conditional probabilities in domain managers' models are randomly generated from range $(0,1)$. We assume that all intradomain path services are observable, i.e., if as a result of a fault a path failure occurs, the corresponding symptom is always observed by the DM. The observability ratio for inter-domain paths is related to the number of network domains. We vary the number of domains between 10 and 25 , using observability ratios of $2 \%$ and $.5 \%$, respectively. We vary the size of network domains from 5 to 25 nodes. Thus the overall experiment covers networks consisting of 50-1250 nodes.

The test scenarios are generated using the belief network model built by the managers. This technique of generating scenarios assumes that the fault propagation model accurately represents relationships among faults and symptoms. Two performance metrics are calculated: detection rate $D R$ defined as a percentage of faults occurring in the network which are isolated by the technique, and false positive rate $F P R$ defined as a percentage of faults reported by the technique that are not occuring in the network. The preliminary results of this simulation study are shown in Table 3

We distinguish three types of experiments, involving: only intra-domain link failures, only inter-domain link failures, and both types of failures. Clearly, the mixed-failure scenarios are the most difficult to diagnose since they always involve at least two concurrent 
Table 3. Simulation study results (DR - detection rate, FPR - false positive rate)

\begin{tabular}{|c|c|c|c|c|c|c|c|}
\hline \multirow{3}{*}{ No. of Domains } & \multirow{3}{*}{$\begin{array}{c}\text { Nodes } \\
\text { per domain }\end{array}$} & \multirow{3}{*}{$\begin{array}{l}\text { Total } \\
\text { nodes }\end{array}$} & \multicolumn{5}{|c|}{ Symptom types } \\
\hline & & & \multirow{2}{*}{\multicolumn{5}{|c|}{ 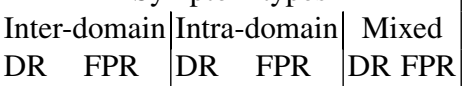 }} \\
\hline & & & & & & & \\
\hline \multirow{5}{*}{10} & 5 & 50 & .80 & .06 & .95 & .04 & $.70 \quad .06$ \\
\hline & 10 & 100 & .90 & .02 & .95 & .04 & $.80 \quad .04$ \\
\hline & 15 & 150 & .95 & .02 & .95 & .04 & $.90 \quad .02$ \\
\hline & 20 & 200 & .90 & .00 & .95 & .02 & $\begin{array}{ll}.85 & .02\end{array}$ \\
\hline & 25 & 250 & .85 & .00 & .90 & .02 & $.70 \quad .02$ \\
\hline \multirow{5}{*}{50} & 5 & 250 & .80 & .06 & .95 & .06 & $\begin{array}{ll}.85 & .06\end{array}$ \\
\hline & 10 & 500 & .95 & .04 & .90 & .06 & $.85 \quad .06$ \\
\hline & 15 & 750 & .95 & .04 & .90 & .04 & $\begin{array}{ll}.75 & .04\end{array}$ \\
\hline & 20 & 1000 & 1.0 & .02 & .85 & .04 & $.70 \quad .02$ \\
\hline & 25 & 1250 & 1.0 & .02 & .85 & .02 & $.65 \quad .02$ \\
\hline
\end{tabular}

faults and the interpretation of their symptoms, which may overlap, leads to ambiguity. Irrespective of the scenario type used, we observe the relationship between the network topology size and the fault localization accuracy achievable with the distributed algorithm. This observation is consistent with the results of the simulation study utilizing the centralized algorithm [17]. This study shows that as the network size grows, as a result of the increasing number of possible failure suspects, the probability of proposing a highly probable, but incorrect or partly correct solution increases.

\section{Related Work}

Many researchers have recognized the importance of distributed fault localization [1,8, 19]. However, few distributed fault localization techniques have actually been proposed. The theoretical foundation for the design of such systems has been laid by Bouloutas et al. [1] and Katzela et al. [8], who investigate different schemes of non-centralized fault localization: decentralized and distributed schemes. The technique proposed in this paper has properties of both these schemes. Similarly to the decentralized scheme [8], we envision a hierarchy of managers with a central manager (GM) making the final fault determination. Unlike in the decentralized scheme [8], however, the GM not only arbitrates among solutions proposed by the domain managers, but also participates in the actual fault determination by proposing its own hypothesis composed of network faults that cannot be identified by the domain managers.

This paper utilizes Pearl's belief updating [12] as an approximation scheme [17]16] in fault localization performed by the managers on all layers of the hierarchy. Other non-deterministic fault localization algorithms could be considered for this purpose: maximum mutual dependency heuristics [9], statistical methods [4], or the incremental algorithm proposed in [14].

Belief networks have previously been applied to the problem of fault diagnosis, but the reported solutions are limited to narrow applications [3 5,18]. These solutions either assume a tree-shaped belief network model [18] or disregard uncertainty involved in 
causal relationships between faults symptoms, i.e., conditional probabilities are 0,1valued [3,5]. The approach proposed in this paper is more general in this respect.

\section{Conclusion and Future Work}

This paper introduces a distributed non-deterministic fault localization algorithm suitable for the diagnosis of end-to-end service problems in communication systems. It builds upon the previously proposed centralized algorithm [17], and increases the admissible network size by an order of magnitude. The algorithm divides the computational effort and system knowledge involved in end-to-end service failure diagnosis among multiple, hierarchically organized managers. The technique is suitable for end-to-end service failure diagnosis in networks with hierarchical topologies.

Future work will involve several important improvements to the proposed technique. The accuracy of the algorithm needs to be increased in scenarios involving mixed types of faults. The performance of the algorithm may be further improved by having the GM delegate the fault diagnosis task to the managers of only those domains that are the most likely to contain a faulty link. The theoretical analysis of signaling overhead of the algorithm is also required. Finally, an extensive simulation study will be conducted 2

\section{References}

1. A. T. Bouloutas, S. B. Calo, A. Finkel, and I. Katzela. Distributed fault identification in telecommunication networks. Journal of Network and Systems Management, 3(3), 1995.

2. G. F. Cooper. Probabilistic inference using belief networks is NP-Hard. Technical Report KSL-87-27, Stanford University, 1988.

3. R. H. Deng, A. A. Lazar, and W. Wang. A probabilistic approach to fault diagnosis in linear lightwave networks. In Hegering and Yemini [6], pp. 697-708.

4. M. Fecko and M. Steinder. Combinatorial designs in multiple faults localization for battlefield networks. In IEEE Military Commun. Conf. (MILCOM), McLean, VA, 2001.

5. D. Heckerman and M. P. Wellman. Bayesian networks. Communications of the ACM, 38(3):27-30, Mar. 1995.

6. H. G. Hegering and Y. Yemini, eds. Integrated Network Management III. North-Holland, Apr. 1993.

7. G. Jakobson and M. D. Weissman. Alarm correlation. IEEE Network, 7(6):52-59, Nov. 1993.

8. I. Katzela, A. T. Bouloutas, and S. Calo. Comparison of distributed fault identification schemes in communication networks. Technical Report RC 19630 (87058), T. J. Watson Research Center, IBM Corp., Sep. 1993.

9. I. Katzela and M. Schwartz. Schemes for fault identification in communication networks. IEEE Transactions on Networking, 3(6):733-764, 1995.

10. S. Kliger, S. Yemini, Y. Yemini, D. Ohsie, and S. Stolfo. A coding approach to event correlation. In Sethi et al. [13], pp. 266-277.

11. A. Medina, A. Lakhina, I. Matta, and J. Byers. BRITE: Universal topology generation from a user's perspective. Technical Report 2001-003, 12001.

\footnotetext{
${ }^{2}$ The views and conclusions contained in this document are those of the authors and should not be interpreted as representing the official policies, either expressed or implied, of the Army Research Laboratory or the U. S. Government.
} 
12. J. Pearl. Probabilistic Reasoning in Intelligent Systems: Networks of Plausible Inference. Morgan Kaufmann Publishers, 1988.

13. A. S. Sethi, F. Faure-Vincent, and Y. Raynaud, eds. Integrated Network Management IV. Chapman and Hall, May 1995.

14. M. Steinder and A. S. Sethi. Non-deterministic diagnosis of end-to-end service failures in a multi-layer communication system. In Proc. of ICCCN, Scottsdale, AZ, 2001. pp. 374-379.

15. M. Steinder and A. S. Sethi. The present and future of event correlation: A need for end-to-end service fault localization. In N. Callaos et al., ed, World Multi-Conf. Systemics, Cybernetics, and Informatics, vol. XII, Orlando, FL, 2001. pp. 124-129.

16. M. Steinder and A. S. Sethi. Increasing robustness of fault localization through analysis of lost, spurious, and positive symptoms. In Proc. of IEEE INFOCOM, New York, NY, 2002.

17. M. Steinder and A.S. Sethi. End-to-end service failure diagnosis using belief networks. In Proc. Network Operation and Management Symposium, Florence, Italy, 2002.

18. C. Wang and M. Schwartz. Identification of faulty links in dynamic-routed networks. Journal on Selected Areas in Communications, 11(3):1449-1460, Dec. 1993.

19. S. A. Yemini, S. Kliger, E. Mozes, Y. Yemini, and D. Ohsie. High speed and robust event correlation. IEEE Communications Magazine, 34(5):82-90, 1996. 